\title{
Suprathermal particles from corotating interaction regions during the first perihelion pass of Solar Orbiter
}

\author{
R. C. Allen ${ }^{1} \odot$, G. M. Mason ${ }^{1}$, G. C. $\mathrm{Ho}^{1}$, J. Rodríguez-Pacheco² ${ }^{2}$, R. F. Wimmer-Schweingruber ${ }^{3}$, G. B. Andrews ${ }^{1}$,
} L. Berger ${ }^{3}$, S. Boden ${ }^{3,4}$, I. Cernuda ${ }^{2}$, F. Espinosa Lara $^{2}$, J. L. Freiherr von Forstner ${ }^{3}$, R. Gómez-Herrero ${ }^{2}$, J. R. Hayes ${ }^{1}$, S. R. Kulkarni ${ }^{3,5}$, W. J. Lees ${ }^{1}$, C. Martin ${ }^{3,6}$, D. Pacheco ${ }^{3}$, O. R. Polo ${ }^{2}$, M. Prieto ${ }^{2}$, A. Ravanbakhsh ${ }^{3,7}$, S. Sánchez-Prieto ${ }^{2}$, C. E. Schlemm ${ }^{1}$, H. Seifert ${ }^{1}$, J. C. Terasa ${ }^{3}$, K. Tyagi ${ }^{1,8}$, Z. Xu ${ }^{3}$, and M. Yedla ${ }^{3,7}$

\footnotetext{
1 Johns Hopkins Applied Physics Lab., Laurel, MD 20723, USA e-mail: Robert.Allen@jhuapl.edu

2 Space Research Group, Universidad de Alcalá, Alcalá de Henares, Madrid, Spain

3 Institut für Experimentelle und Angewande Physik, Christian-Albrechts-Universität zu Kiel, Kiel, Germany

${ }^{4}$ Now at DSI Datensicherheit GmbH, Rodendamm 34, 28816 Stuhr, Germany

5 Now at Deutsches Elektronen-Synchrotron (DESY), Platanenallee 6, 15738 Zeuthen, Germany

${ }^{6}$ Now at German Aerospace Center (DLR), Department of Extrasolar Planets and Atmospheres, Berlin, Germany

7 Now at Max-Planck-Institute for Solar System Research, Justus-von-Liebig-Weg 3, 37077 Göttingen, Germany

8 Now at Univ. Colorado/LASP, Boulder, CO, USA
}

Received 6 November 2020 / Accepted 20 December 2020

\section{ABSTRACT}

\begin{abstract}
The first orbit of Solar Orbiter provided comprehensive measurements of six corotating interaction regions (CIRs) within 1 au. Five of these CIRs were also observed by ACE at 1 au, allowing for comparisons of the suprathermal ion intensities and spectra at different radial distances. Only subtle modulations of the ${ }^{4} \mathrm{He}$ spectral slopes are observed between Solar Orbiter and ACE. Additionally, the radial gradients of $226-320 \mathrm{keV} / \mathrm{nuc}^{4} \mathrm{He}$ ion intensities between Solar Orbiter and ACE are similar to that of $1.53 \mathrm{MeV} \mathrm{H}$ reported by Van Hollebeke et al. (1978, J. Geophys. Res., 83, A10). These observations provide a new addition to the study of the radial dependence of CIR-associated suprathermal ions in the inner heliosphere.
\end{abstract}

Key words. acceleration of particles - solar wind - Sun: abundances - interplanetary medium - shock waves

\section{Introduction}

Corotating interaction regions (CIRs) occur along the boundary where high-speed streams, emanating from coronal holes, interact with preceding slower solar wind. These persistent, large-scale structures are often associated with energetic particles that are accelerated by shocks and typically located beyond 1 au (Richardson 2004, and references therein); however, recent studies suggest that additional acceleration could be a result of compressive acceleration along the CIR interface prior to the formation of shocks at the CIR boundaries (e.g., Chotoo et al. 2000; Giacalone et al. 2002; Chen et al. 2015). While the particles are associated with the CIR structures, the associated particle enhancements are not necessarily colocated with the bulk structure, but may lie on magnetic field lines that are connected to the region along the structure that is accelerating the ions. As a result, CIR-associated particle enhancements can often be observed up to several days following the passage of a CIR (Balogh et al. 1999; Richardson 2018, and references therein).

Radial gradients in the intensity of CIR-associated energetic particles have been reported in the past (e.g., Kunow et al. 1977; Van Hollebeke et al. 1978; Christon 1981). Van Hollebeke et al. (1978) examined energetic particle acceleration and transport by comparing CIR-associated energetic (0.96-2.2 MeV) proton peak intensities observed by Helios 1 and 2 ( 0.3 to $1 \mathrm{au})$,
Interplanetary Monitoring Platform (IMP) 7 (1 au), Pioneer 11 (3.8 au), and Pioneer 10 (9 to $10 \mathrm{au}$ ) during the declining phase of solar cycle 20 (events spanning from mid-1973 to mid-1976). These comparisons revealed that, from 0.3 to $3 \mathrm{au}$, the peak intensity of energetic protons increases with increasing heliocentric distance before decreasing out to $10 \mathrm{au}$. This finding, along with observations of sunward streaming ions, suggests particle acceleration beyond 1 au (Richardson 2004, and references therein). While the events presented by Van Hollebeke et al. (1978) are well ordered in radial distance, additional events are required to further investigate the spatial and temporal variations of CIR-associated suprathermal and energetic particles. For example, reoccurring observations of the same CIR can have drastically different suprathermal ion fluxes (e.g., Allen et al. 2021), and the relative abundance of suprathermal ions associated with CIRs are known to have a solar cycle variation (e.g., Mason et al. 2008, 2012; Allen et al. 2019). The addition of Solar Orbiter to the current heliospheric fleet of observatories allows for additional conjunctive observations to expand upon the studies of CIR-associated suprathermal ion populations.

Initial observations by Parker Solar Probe (PSP) of inner heliospheric CIRs and CIR-associated energetic ions have recently been reported (e.g., McComas et al. 2019; Allen et al. 2020a,c, 2021; Cohen et al. 2020; Desai et al. 2020; Joyce et al. 2020, 2021). One such finding is that the CIR-associated 


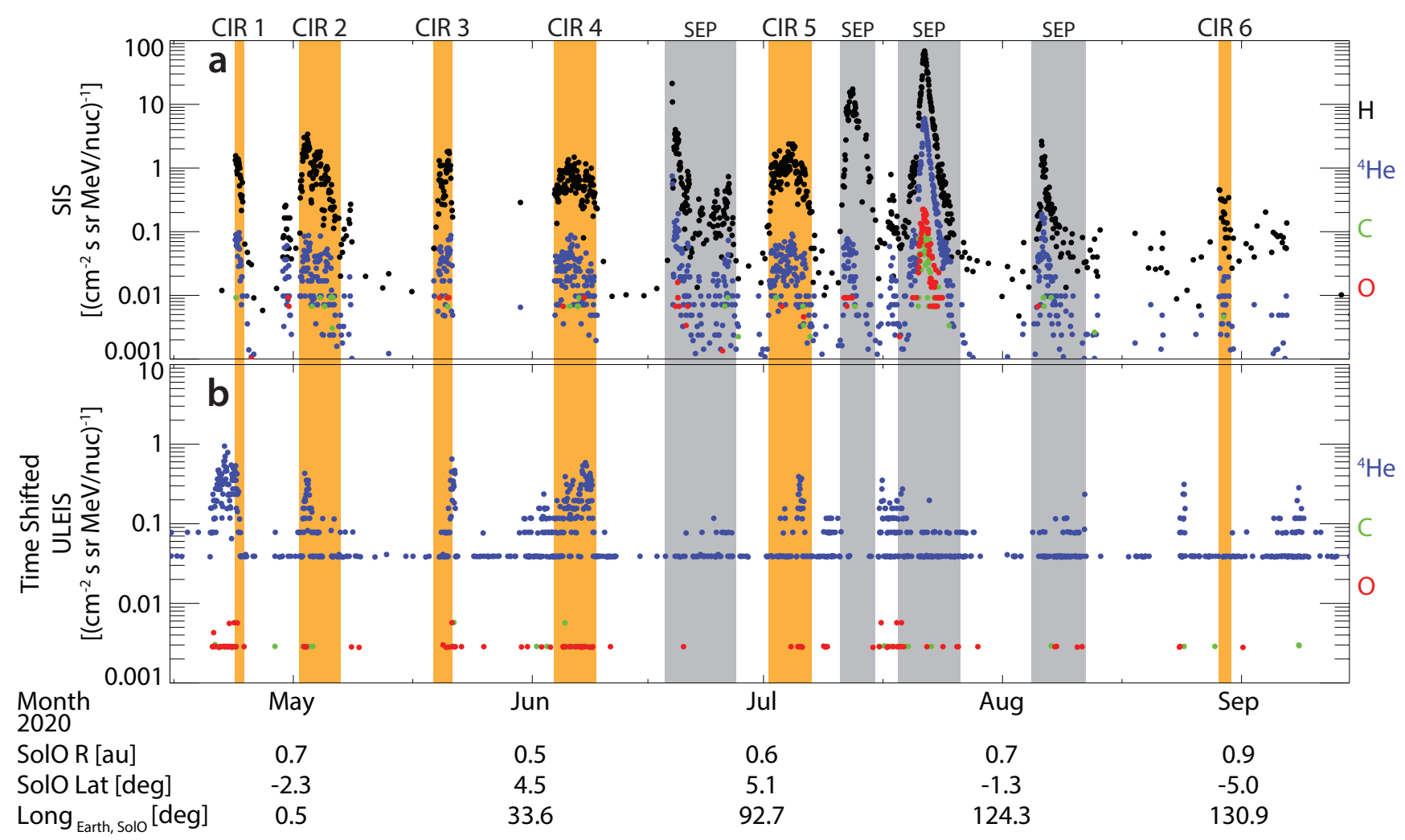

Fig. 1. Overview of 226-320 keV/nuc Solar Orbiter-SIS and ACE-ULEIS observations from 2020 April 15 until 2020 Sept. 15. The ULEIS observations are time-shifted to the SIS observation times as described in the text. The six observed CIR-associated particle events are indicated by the orange-shaded regions, and the four SEP events during this time frame are marked by the gray boxes.

particle spectra observed by PSP (e.g., Joyce et al. 2021; Schwadron et al. 2021; Allen et al. 2021) display little to no modulation with radial distance, which is consistent with earlier observations (e.g., Christon 1981). This is in conflict with the model developed by Fisk \& Lee (1980), which predicts that the higher-energy portion of the of the spectra will soften as the particles move into the inner heliosphere, while the lowerenergy spectra will harden and eventually roll over. To better explain the absence of this feature in the PSP-observed spectra, Schwadron et al. (2021) demonstrated that sub-Parker spiral field configurations may allow for more direct access of the CIRassociated ions into the inner heliosphere, lessening the transport effects predicted by Fisk \& Lee (1980). Sub-Parker spiral field lines are a result of the motion of magnetic footpoints across the coronal hole boundary, leading to a more radial, or less wound, field line topology than the normal Parker spiral (e.g., Murphy et al. 2002; Schwadron 2002; Schwadron \& McComas 2005; Schwadron et al. 2021). Additional observations, particularly those within 1 au for comparison, are needed to further investigate the energization and transport effects of CIRassociated ions. Such observations, provided by the recently launched Solar Orbiter mission, are the focus of this Letter.

\section{Missions and datasets}

The Solar Orbiter mission (Müller et al. 2020) was launched in February 2020 to better understand the physical processes occurring on the Sun and in interplanetary space. The first perihelion of Solar Orbiter reached a heliocentric distance of 0.52 au on 15 June 2020. Measurements of suprathermal and energetic solar wind particles on Solar Orbiter are made by the Energetic Particle Detector (EPD) suite (Rodríguez-Pacheco et al. 2020).
This study incorporates observations from the Suprathermal Ion Spectrograph (SIS), part of the EPD suite, which measures the suprathermal ion composition of $\mathrm{H}$ through $\mathrm{Fe}$ ions in the energy range $0.1-10 \mathrm{MeV} /$ nuc. The SIS, a time-of-flight-based instrument with a solid-state detector at the end of the flight path, consists of two identical telescopes that look roughly along the Parker spiral toward and away from the sun. The telescope count rates are combined into a single product for this analysis, which is appropriate due to the typically low anisotropy of CIR events. After SIS had 2 months to outgas, the high voltage was raised and operations began in April 2020.

To allow for investigations of the radial evolution of the ions, we used observations taken by the Ultra-Low Energy Isotope Spectrometer (ULEIS, Mason et al. 1998) on board the Advanced Composition Explorer (ACE, Stone et al. 1998) spacecraft, which launched in 1997 and has since been orbiting the sunward Sun-Earth Lagrange point L1, at roughly $1 \mathrm{au}$. The design of ULEIS is similar to SIS, with ULEIS measuring He to $\mathrm{Ni}$ ions with energies between $45 \mathrm{keV} /$ nuc and a few $\mathrm{MeV} /$ nuc. However, due to the low ULEIS detection efficiency for $\mathrm{H}$, only ${ }^{4} \mathrm{He}$ ions observed by ULEIS are presented here. Future investigations will include more detailed composition comparisons between ULEIS and SIS.

\section{Results}

An overview of $226-320 \mathrm{keV} / \mathrm{nuc}$ ions $\left(\mathrm{H},{ }^{4} \mathrm{He}, \mathrm{C}\right.$, and $\mathrm{O}$ ) observed from 2020 April 15 through 2020 September 15 by SIS are shown in Fig. 1a. During this time frame, six CIRassociated suprathermal particle events were seen (orange time periods) along with four solar energetic particle (SEP) events (gray time periods). The SEP events are discussed in more detail 

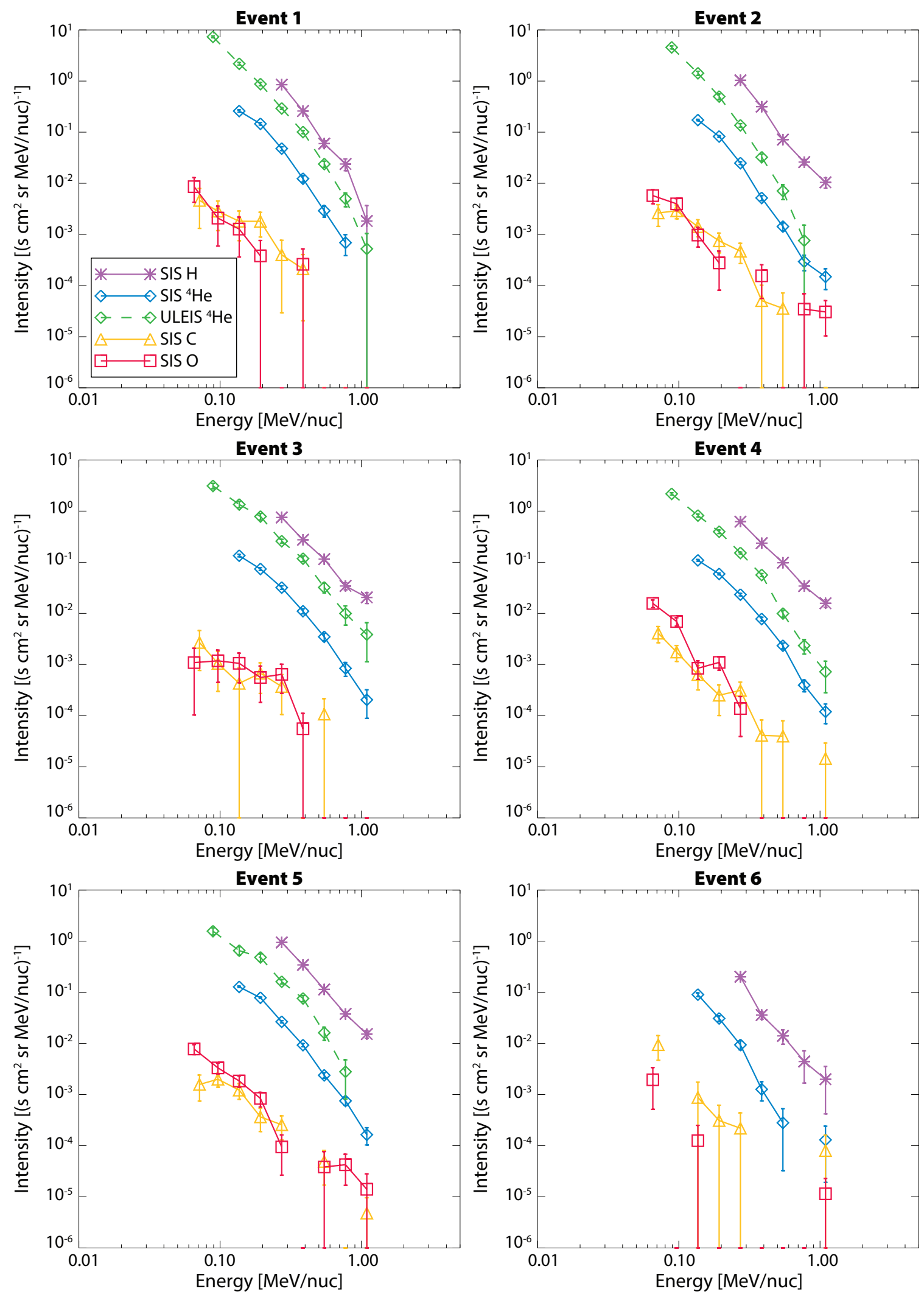

Fig. 2. Ion spectra for $\mathrm{H},{ }^{4} \mathrm{He}, \mathrm{C}$, and $\mathrm{O}$ from SIS and ${ }^{4} \mathrm{He}$ from ULEIS with energies less than $1.5 \mathrm{MeV} /$ nuc for the CIR-associated events. CIR-associated event 6 did not have a conjugate ULEIS observation.

in Mason et al. (2021) and Gómez-Herrero et al. (2021). To better compare the CIR-associated events (henceforth referred to as numbered "CIR events") from SIS and ULEIS, estimated time lags were computed between ACE and Solar Orbiter. To compute these lags, the Parker spiral footpoints for each spacecraft were computed in Carrington longitudes for each data point assuming $300 \mathrm{~km} \mathrm{~s}^{-1}$ solar wind, consistent with observations at ACE during this time period. Applying a fixed corotation 
Table 1. CIR-associated energetic particle event properties.

\begin{tabular}{|c|c|c|c|c|c|c|c|}
\hline & Event & 1 & 2 & 3 & 4 & 5 & 6 \\
\hline Solar & Start time ${ }^{(a)}$ & 23 Apr. (114) & 1 May (122) & 19 May (140) & 3 Jun. (155) & 1 Jul. (183) & 29 Aug. (242) \\
\hline Orbiter & & $10: 00^{(e)}$ & 20:00 & 04:00 & 20:00 & 11:00 & 02:00 \\
\hline \multirow[t]{5}{*}{ SIS } & End time $(a)$ & 24 Apr. (115) & 7 May (128) & 21 May (142) & 9 Jun. (161) & 7 Jul. (189) & 30 Aug. (243) \\
\hline & & $21: 00$ & 03:00 & $16: 00$ & 10:00 & 19:00 & 19:00 \\
\hline & Distance $(\mathrm{au})^{(b)}$ & 0.78 & 0.73 & 0.62 & 0.54 & 0.56 & 0.89 \\
\hline & H spectral index $(c)$ & -3.5 & -3.6 & -2.9 & -2.8 & -3.1 & -3.6 \\
\hline & ${ }^{4} \mathrm{He}$ spectral index $(c)$ & -4.1 & -4.2 & -3.5 & -3.9 & -3.5 & -5.1 \\
\hline \multirow{5}{*}{$\begin{array}{l}\mathrm{ACE} \\
\text { ULEIS }\end{array}$} & Start time ${ }^{(a)}$ & 21 Apr. (112) & 3 May (124) & 21 May (142) & 1 Jun. (153) & 30 Jun. (182) & $-{ }^{(d)}$ \\
\hline & & 07:00 & 00:00 & $15: 00$ & 19:00 & $19: 00$ & \\
\hline & End time ${ }^{(a)}$ & 24 Apr. (115) & 4 May (125) & 22 May (143) & 8 Jun. (129) & 1 Jul. (183) & $-(d)$ \\
\hline & & $12: 00$ & $22: 00$ & $12: 00$ & 00:00 & 20:00 & \\
\hline & ${ }^{4}$ He spectral index $(c)$ & -3.9 & -4.9 & -3.2 & -4.1 & -4.0 & $-(d)$ \\
\hline
\end{tabular}

Notes. ${ }^{(a)}$ Year: 2020, with day of year in parentheses. ${ }^{(b)}$ Solar Orbiter heliocentric distance (au). ${ }^{(c)}$ In energy, over the energy range $0.2-1 \mathrm{MeV} /$ nuc. ${ }^{(d)}$ No corresponding ULEIS observation. ${ }^{(e)}$ Start time for event 1 was impacted by the SIS high-voltage ramp-up.

speed $\left(14.7^{\circ} \mathrm{day}^{-1}\right)$, the differences in these longitudes were used to estimate the time between observations at ACE and Solar Orbiter. While the true corotation speed of CIRs has been observed to have some variation (Allen et al. 2020b), varying the corotation speed did not significantly affect this shift. This timeshift was used to adjust the ACE-ULEIS observations into the time frame of Solar Orbiter (Fig. 1b) and varied from 0 days at CIR event 1 to 5 days for CIR event 5. Comparing solar wind properties at L1 (not shown), CIR events 1 and 3 are associated with the same CIR structure, while CIR events 2, 4, 5, and 6 are associated with a separate CIR structure. As shown in Fig. 1, CIR events $1-5$ are all observed by both SIS and ULEIS. In addition to being located adjacent to a CIR structure identified by ACE, the events also display normal features of a CIR-associated particle event (e.g., dispersionless flat-topped enhancement lasting $\gtrsim 1$ day, corresponding observations between SIS and ULEIS when factoring in corotation, expected ion composition, and low anisotropy between the two SIS telescopes). The SIS sensor was undergoing high-voltage ramp-up procedures for initial turn-on during the first CIR event, and so there are only SIS observations for the end of the event, limiting robust comparisons to ACE. The small enhancement on 17 July may be associated with a CIR; however, the proximity to the SEP events limits the ability to cleanly analyze the event. CIR event 6 was not observed by ULEIS, possibly due to it being the weakest of the CIR events observed by Solar Orbiter or due to differences in latitude.

Figure 2 illustrates average ion spectra for each event using the start and stop times listed in Table 1. Slopes of computed power-law-in-energy fits for $0.2-1 \mathrm{MeV} /$ nuc $\mathrm{H}$ and ${ }^{4} \mathrm{He}$ ions are also given in Table 1. For comparison, the ACE-ULEIS ${ }^{4} \mathrm{He}$ spectra are shown alongside the SIS spectra, with power-law fits, computed the same way as those for SIS, listed in Table 1. Comparing the ${ }^{4} \mathrm{He}$ spectra from ACE and Solar Orbiter, the spectral slopes show general agreement. The much lower intensities observed by SIS compared to ULEIS (Fig. 2 and Table 1) suggests only low amounts of energy-dependent modulation due to transport processes (i.e., the spectral hardening of lower-energy ions as predicted by Fisk \& Lee 1980 is not observed). The abundance and spectral slopes of $\mathrm{C}$ and $\mathrm{O}$ ions at Solar Orbiter are similar to each other, consistent with prior observations of CIRassociated suprathermal ions at 1 au (e.g., Mason et al. 2008). For each species, the spectra are very similar between all of the events. The spectral power-law exponents of the $\mathrm{H}$ and ${ }^{4} \mathrm{He}$ ions

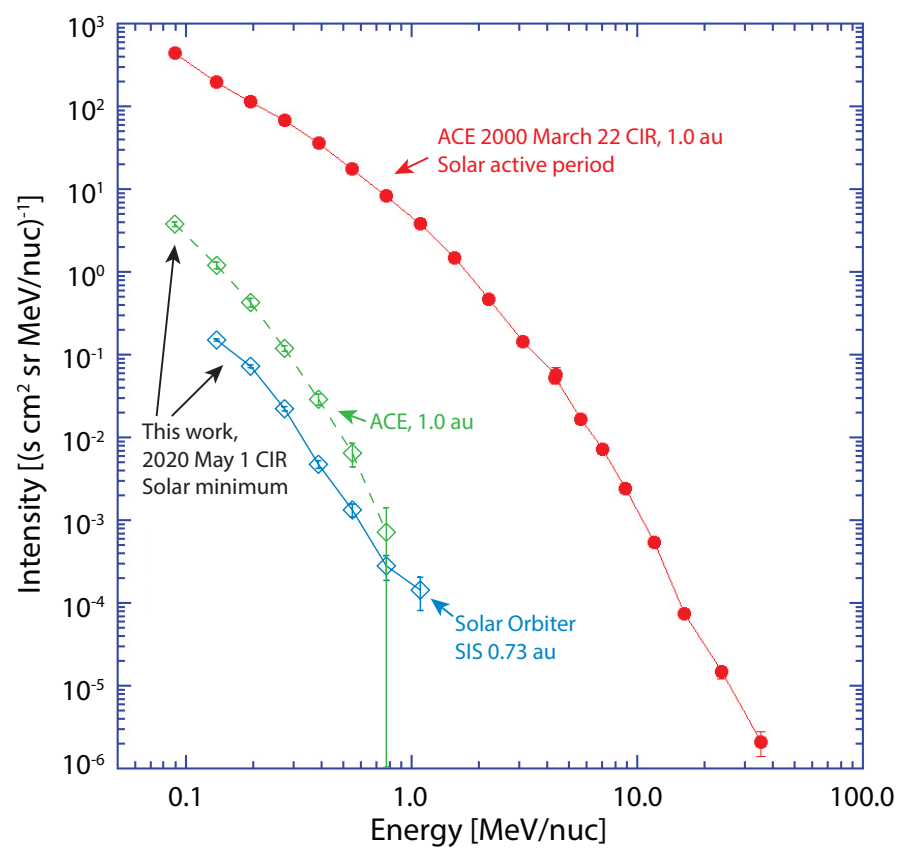

Fig. 3. ${ }^{4} \mathrm{He}$ spectra for event 2 shown with a representative CIR event at ACE in 2000.

(Fig. 2 and Table 1) are very similar, with the $\mathrm{H}$ spectrum always slightly harder than that for ${ }^{4} \mathrm{He}$, similar to previous observations (e.g., Richardson et al. 1993; Kunow et al. 1977). While the CIR event 1 comparison to ACE is similar to the other events, care must be taken when interpreting this event as the SIS instrument became operational during the event.

As seen in Fig. 2, the CIR-associated particle enhancements observed by SIS have very low intensities, with spectra falling below detectability around $1 \mathrm{MeV} /$ nuc. By comparison, Mason et al. (2008) surveyed 41 CIRs observed by ACE at 1 au between 1998 and 2007, with peak ${ }^{4} \mathrm{He}$ intensities ranging from $\sim 20$ to 3000 times larger than those observed at ACE in Fig. 1. Figure 3 compares the ${ }^{4} \mathrm{He}$ spectra for CIR 2 at Solar Orbiter and ACE with a CIR observed by ACE on 22 March 2000, a representative event reported in Mason et al. (2008). Not only is the 22 March 2000 CIR observed at ACE much more intense than the SIS-observed CIR, but the spectrum below $1 \mathrm{MeV} /$ nuc 


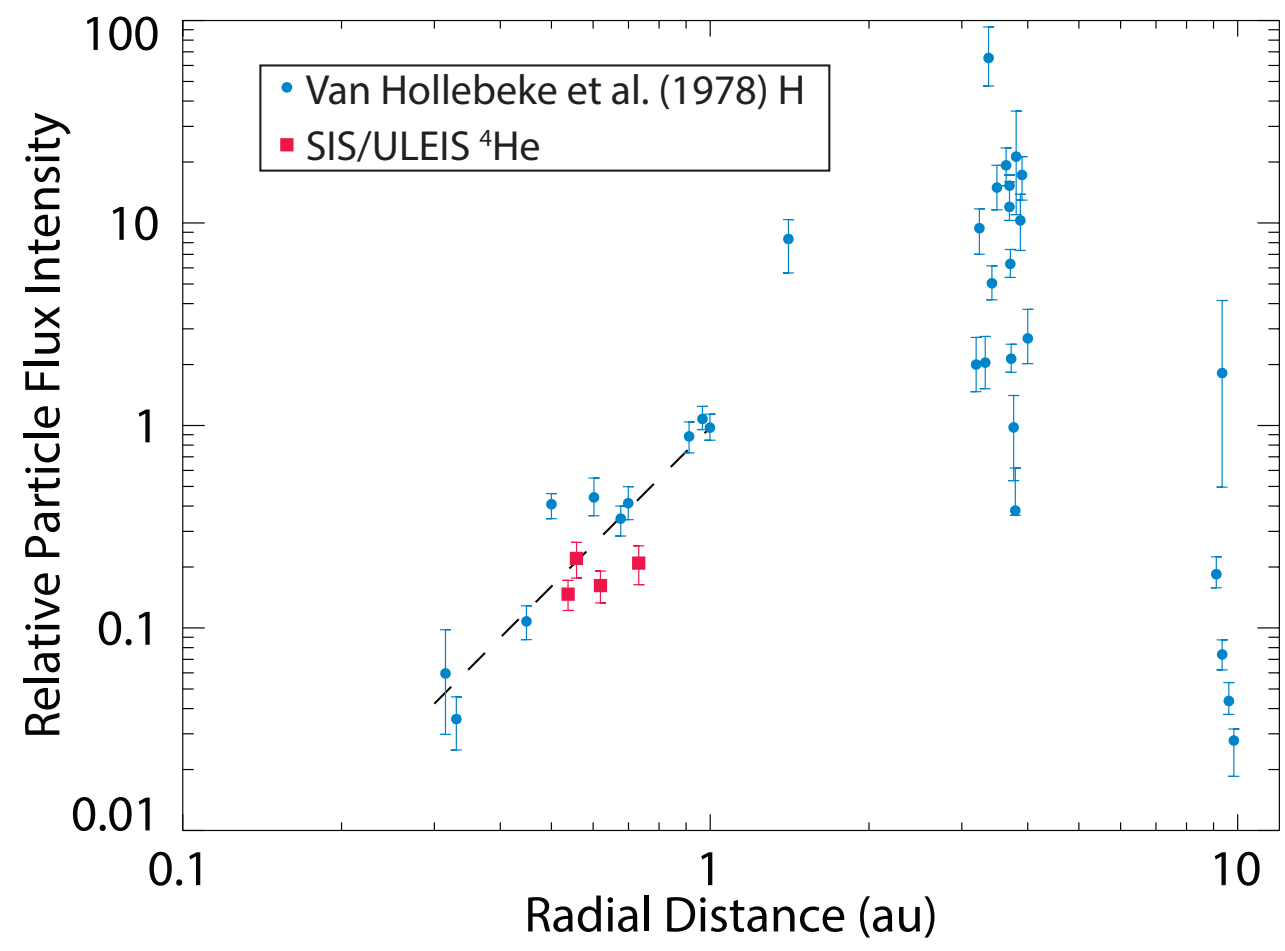

Fig. 4. Relative particle flux intensity (normalized to intensities at $1 \mathrm{au}$ ) as a function of radial distance from both SIS-ULEIS (red, 226-320 keV/nuc $\left.{ }^{4} \mathrm{He}\right)$ and from Van Hollebeke et al. (1978) (blue, $0.9-2.2 \mathrm{MeV} \mathrm{H}$ ). The dashed line is included as a visual guide for comparing data points in the inner heliosphere. is also harder than that observed in the CIR 2 event of this Letter. The CIR-associated energetic particle events observed by both ACE and Solar Orbiter in this study have spectral slopes similar to those of particles at energies beyond the few MeV/nuc knee in the spectra for more intense CIR events (Richardson 2004, and references therein). There are several possibilities that could explain these differences. During 2020, the solar wind speed observed on ACE was low, below $300 \mathrm{~km} \mathrm{~s}^{-1}$ during lowspeed streams, and only increased to $400-500 \mathrm{~km} \mathrm{~s}^{-1}$ during the high-speed streams associated with CIRs 2-5 in Fig. 1. If the CIRs observed by ACE and Solar Orbiter are created by local compressions, the low speeds might be the cause of the lower intensity. There is also the possibility that differences in the suprathermal ion pool between solar active and solar minimum periods affect the seed population and thereby the accelerated particles observed in the CIRs (e.g., Chotoo et al. 2000; Desai et al. 2006; Mason et al. 2012; Allen et al. 2019).

To better investigate how the abundance of ${ }^{4} \mathrm{He}$ varies as a function of radial distance, a six-hour average around the peak intensity was taken for events $2-5$ for SIS and ULEIS. Event 1 was not included in this analysis due to ambiguity of whether or not SIS would have observed the peak intensity due to its high-voltage turn-on. Event 6 was excluded due to the lack of a conjugate observation at ACE. The resulting SIS ${ }^{4} \mathrm{He}$ intensities, normalized to 1 au by dividing the SIS intensities by the ULEIS intensities, are shown in Fig. 4 (red points) as a function of radial distance. The blue points in Fig. 4 are the normalized 0.9 to $2.2 \mathrm{MeV} \mathrm{H}$ intensities from Van Hollebeke et al. (1978), and the dashed line is included as a visual guide for this comparison. Despite having different energies and comprising heavy ions, the radial gradients in the ${ }^{4} \mathrm{He}$ intensities between SIS and ULEIS are very similar to those found for more energetic $\mathrm{H}$ between Helios 1 and 2 and IMP 7, although at slightly lower relative intensities. This is particularly curious as $\mathrm{H}$ and ${ }^{4} \mathrm{He}$ ions have different rigidities, which should lead to different transport properties. More observations are needed to better determine if the normalized ${ }^{4} \mathrm{He}$ ion intensities are generally lower than the normalized energetic $\mathrm{H}$, particularly given the fact that these are different species and energies and are from different solar cycles.

\section{Discussion and conclusions}

Six CIR-associated suprathermal ion enhancements within 1 au were observed during the first orbit of Solar Orbiter. Five of these events have corresponding observations at ACE, allowing for an initial investigation of the radial evolution of associated suprathermal ions. These suprathermal ions show only subtle variations in the ${ }^{4} \mathrm{He}$ spectral slopes compared to observations at $1 \mathrm{au}$, consistent with recent PSP observations that investigated transport effects on inner heliospheric CIR-associated particle spectra (Joyce et al. 2021; Allen et al. 2021). Additionally, the particle spectral slopes are similar to previous observations (e.g., Richardson et al. 1993; Kunow et al. 1977), and, as previously observed at 1 au (Richardson 2018, and references therein), the SIS-observed $\mathrm{C}$ and $\mathrm{O}$ ion spectra are very similar to one another. Comparing the ratio of $226-320 \mathrm{keV} / \mathrm{nuc}{ }^{4} \mathrm{He}$ ion intensities from SIS at 0.5 au normalized to those observed at $1 \mathrm{au}$ by ULEIS (Fig. 4), the relative intensity is seen to scale with heliocentric distance very similarly to the 0.9 to 2.2 MeV H intensities reported by Van Hollebeke et al. (1978) and Christon \& Simpson (1979), despite being different species, having different energies, and occurring at different solar cycle phases. Future observations of additional CIRs at various heliocentric distances, along with other species observed by both SIS and ULEIS, will allow for a more in-depth study of potential mass or mass-per-charge effects on ion transport and scattering in the inner heliosphere. Finally, longer-term investigations between ACE and Solar Orbiter, as well as comparisons to previous Ulysses observations, may also provide insight into latitudinal variations of the suprathermal ion intensities associated with CIRs when Solar Orbiter leaves the ecliptic plane.

Acknowledgements. The Suprathermal Ion Spectrograph (SIS) is a European facility instrument funded by ESA. The SIS instrument was constructed by the JHU/Applied Physics Lab, with assistance from CAU Kiel. We thank the 
many individuals at ESA and within the Energetic Particle Detector team for their support in its development. Post launch operation of SIS is funded by NASA contract NNN06AA01C, and we thank NASA headquarters and the NASA/GSFC Solar Orbiter project office for their continuing support. ACE ULEIS is supported by NASA grant NNX17AC05G. The UAH team acknowledges the financial support by the Spanish Ministerio de Ciencia, Innovación y Universidades FEDER/MCIU/AEI Projects ESP2017-88436-R and PID2019104863RB-I00/AEI/10.13039/501100011033. The CAU Kiel team thanks ESA for supporting the build of SIS under contract number SOL.ASTR.CON.00004; the German Federal Ministry for Economic Affairs and Energy and the German Space Agency (Deutsches Zentrum für Luft- und Raumfahrt, e.V., (DLR)) for their unwavering support under grant numbers 50OT0901, 50OT1202, 50OT1702, and 50OT2002; and the University of Kiel and the Land SchleswigHolstein for their support of SIS. We acknowledge solar wind data from the ACE Science Center website.

\section{References}

Allen, R. C., Ho, G. C., \& Mason, G. M. 2019, ApJ, 883, L10

Allen, R. C., Lario, D., Odstrcil, D., et al. 2020a, ApJS, 246, 36

Allen, R. C., Ho, G. C., Jian, L. K., et al. 2020b, Space Weather, 18, 1

Allen, R. C., Ho, G. C., Jian, L. K., et al. 2020c, A\&A, 650, A25

Allen, R. C., Ho, G. C., Mason, G. M., et al. 2021, Geophys. Res. Lett., 48 e91376

Balogh, A., Gosling, J. T., Jokipii, J., Kallenbach, R., \& Kunow, H. 1999, Space Sci. Rev., 7, 1

Chen, J. H., Schwadron, N. A., Möbius, E., \& Gorby, M. 2015, J. Geophys. Res. Space Phys., 120, 9269

Chotoo, K., Schwadron, N. A., Mason, G. M., et al. 2000, J. Geophys. Res., 105 A10

Christon, S. P. 1981, J. Geophys. Res., 86, 8852

Christon, S. P., \& Simpson, J. A. 1979, ApJ, 227, L49
Cohen, C. M. S., Christian, E. R., Cummings, A. C., et al. 2020, ApJS, 246, 20

Desai, M. I., Mason, G. M., Mazur, J. E., \& Dwyer, J. R. 2006, ApJ, 645, L81 Desai, M. I., Mitchell, D. G., Szalay, J. R., et al. 2020, ApJS, 246, 56

Fisk, L. A., \& Lee, M. A. 1980, ApJ, 237, 620

Giacalone, J., Jokipii, J. R., \& Kóta, J. 2002, ApJ, 573, 845

Gómez-Herrero, R., Pacheco, D., Kollhoff, A., et al. 2021, A\&A, 656, L3 (SO Cruise Phase SI)

Joyce, C. J., McComas, D. J., Christian, E. R., et al. 2020, ApJS, 246, 41

Joyce, C. J., McComas, D. J., Schwadron, N. A., et al. 2021, A\&A, 651, A2

Kunow, H., Witte, M., Wibberenz, G., et al. 1977, J. Geophys., 42, 615

Mason, G. M., Gold, R. E., Krimigis, S. M., et al. 1998, Space Sci. Rev., 86, 409

Mason, G. M., Leske, R. A., Desai, M. I., et al. 2008, ApJ, 678, 1458

Mason, G. M., Desai, M. I., \& Li, G. 2012, ApJ, 748, L31

Mason, G. M., Ho, G. C., Allen, R. C., et al. 2021, A\&A, 656, L1 (SO Cruise Phase SI)

McComas, D. J., Christian, E. R., Cohen, C. M. S., et al. 2019, Nature, 576, 223 Müller, D., St. Cyr, O. C., Zouganelis, I., Gilbert, H. E., \& Marsden, R. 2020, A\&A, 642, A1

Murphy, N., Smith, E. J., \& Schwadron, N. A. 2002, Geophys. Res. Lett., 29, 22

Richardson, I. G. 2004, Space Sci. Rev., 111, 267

Richardson, I. G. 2018, Liv. Rev. Sol. Phys., 15, 1

Richardson, I. G., Barbier, L. M., Reames, D. V., \& von Rosenvinge, T. T. 1993, J. Geophys. Res., 98, A1

Rodríguez-Pacheco, J., Wimmer-Schweingruber, R. F., Mason, G. M., et al. 2020, A\&A, 642, A7

Schwadron, N. A. 2002, Geophys. Res. Lett., 29, 14

Schwadron, N. A., \& McComas, D. J. 2005, Geophys. Res. Lett., 32, 1

Schwadron, N. A., Joyce, C. J., Aly, A., et al. 2021, A\&A, 650, A24

Stone, E. C., Frandsen, A. M., Mewaldt, R. A., et al. 1998, Space Sci. Rev., 86, 1

Van Hollebeke, M. A. I., McDonald, F. B., Trainor, J. H., \& von Rosenvinge, T. T. 1978, J. Geophys. Res., 83, A10 